\title{
Análise de similaridade das árvores de Cedrela sp. sob diferentes condições de crescimento no leste do estado do Acre, Brasil
}

\author{
Similarity analysis of Cedrela sp. trees under different \\ growth conditions in eastern state of Acre, Brazil
}

\author{
Moisés Silveira Lobão ${ }^{1}$, Givanildo Pereira Ortega ${ }^{1}$, Edson Amaral ${ }^{2}$, \\ Patrícia Gomes Ribeiro Amorim ${ }^{1}$, Marco Antonio Amaro', \\ Fidel Alejandro Roig ${ }^{3}$ e Mario Tomazello Filho ${ }^{4}$
}

\section{Resumo}

A manutenção da dinâmica da biodiversidade em florestas tropicais depende de certo nível de perturbação ambiental, como a queda natural das árvores proporcionando a abertura de clareiras, a germinação das sementes e o crescimento e desenvolvimento das plantas. Por outro lado, o manejo florestal intervém no ciclo natural das florestas pelo corte e retirada das árvores das espécies de maior volume de madeira e de valor comercial. Porém, ainda há pouco conhecimento sobre o crescimento e a regeneração natural das árvores das centenas de espécies tropicais, o que se constitui em uma lacuna e obstáculo para a aplicação do manejo florestal sustentado e, assim, permitindo a manutenção da biodiversidade. Nesse aspecto, esse trabalho visa determinar a idade e a periodicidade do crescimento do tronco do lenho das árvores de Cedrela sp. no estado do Acre, em diferentes condições de crescimento (sítios e microsítios) para propiciar os subsídios ao manejo florestal sustentável. As árvores de Cedrela sp foram selecionadas em três sítios: Reserva Experimental Catuaba (REC) (com três microssítios), Fazenda São Jorge (F. S. Jorge) e Parque Zoobotânico (PZ). Do DAP do tronco das árvores foram retiradas amostras do lenho para as análises dendrocronológicas. Na seção transversal dessas amostras do lenho foi realizada a demarcação, contagem e a mensuração da largura dos anéis de crescimento. Em seguida, utilizando-se o programa Cofecha foi feito o controle da qualidade das séries dos anéis de crescimento. Com os parâmetros: número, largura média do anel de crescimento, desvio padrão e o DAP do tronco das árvores foi avaliada a similaridade das árvores de Cedrela sp dos sítios e microssítios, através da análise multivariada. Os resultados indicaram que as condições de crescimento específicas dos sítios e dos ambientes influenciaram significativamente no crescimento radial do tronco das árvores de Cedrela sp demonstrando o potencial da dendrocronologia como ferramenta no manejo florestal.

Palavras-chave: Ciclo de corte; Anéis de crescimento; Dendrocronologia.

\begin{abstract}
The biodiversity of tropical forests depends on certain level of environmental disturbances, such as the natural fall of trees providing clearings, seed germination, growth and development of plants that environment. On the other hand, forest management intervenes in this natural cycle, by removing larger trees of the species which are of great commercial value. However, little is known about natural growth and regeneration of the hundreds of tropical species; and this constitutes a gap and obstacle to the implementation of sustainable forest management, by permitting the maintenance of biodiversity. In this regard, this study aims to determine the age and growth periodicity of stem of native Cedrela sp. trees in Acre under different growth conditions (sites and microsites) to provide subsidies to sustainable forest management in this region. Trees of Cedrela sp were selected at three sites: Experimental Reserve Catuaba (REC) (three microsites), Farm São Jorge (FSJ) Zoobotanic Park (PZ). Wood samples were collected at DBH of the trunk for the dendrochronological analysis. This was followed by the demarcation, counting and measuring the width of growth rings. Next, the Cofecha program was used for the quality control of the series of growth rings. Using the parameters: number, average width of the growth ring, standard deviation and the DBH of the tree stems, a similarity of trees Cedrela sp. in site and microsites was observed by multivariate statistical
\end{abstract}

\footnotetext{
1Professor(a) Doutor(a). UFAC - Universidade Federal do Acre. Caixa Postal: 500-69915-900 - Rio Branco, AC, Brasil. E-mail: moises lobao@yahoo.com.br; givaortega@hotmail.com; ribeiropg@unb.br; marcoantonioamaro@hotmail.com ${ }^{2}$ Engenheiro Florestal. ICMBio - Instituto Chico Mendes de Conservação da Biodiversidade. EQSW 103/104 - Bloco C 70.670-350 - Brasília, DF, Brasil. E-mail: edson.amaral@icmbio.gov.br

32iretor. Instituto Argentino de Nivología y Glaciología / Departamento de Dendrocronologia e Historia Ambiental. Caixa Postal: 5500-Mendoza, Argentina. E-mail: froig@mendoza-conicet.gob.ar

${ }^{4}$ Professor Titular. Departamento de Ciências Florestais. USP - Universidade de São Paulo/ESALQ - Escola Superior de Agricultura “Luiz Queiroz". Caixa Postal 09 - 13418-900 - Piracicaba, SP, Brasil. E-mail: mtomazel@usp.br
}

Sci. For., Piracicaba, v. 44, n. 109, p. 231-239, mar. 2016 DOI: dx.doi.org/10.18671/scifor.v44n109.22 
analysis.Different growth conditions (sites and environments) significantly affected the radial growth of trees Cedrela sp., demonstrating the potential of dendrochronology as a tool for forest management.

Keywords: Logging cycle; Tree rings; Dendrochronology.

\section{INTRODUÇÃO}

As florestas tropicais do mundo ocupam apenas 7\% da superfície terrestre sendo que, no entanto, abrigam mais da metade da riqueza das espécies (KATTAN, 2002). Este importante ecossistema do planeta é dinâmico e dependente da polinização, disseminação e germinação das sementes das espécies arbóreas, bem como da morte e queda natural das grandes árvores que promovem a abertura do dossel da floresta para manter a sua dinâmica e biodiversidade (ASQUITH, 2002).

Por isso, a dinâmica e a complexidade dos ecossistemas florestas tropicais precisam ser compreendidos para a sua utilização racional e sustentada. Nesse aspecto, várias áreas do conhecimento científico como a ecologia, fenologia, botânica, climatologia, fisiologia e suas áreas correlatas, são fundamentais, no fornecimento de subsídios visando a manutenção da biodiversidade, a utilização de práticas de manejo florestal sustentável, a avaliação do ciclo do carbono e do efeito do aquecimento global, etc.

Bush (1994); Colinvaux et al. (1996) afirmaram que um nível intermédio de perturbação (p. ex. a queda de uma árvore na floresta com abertura de clareira) favorece a regeneração e o crescimento das árvores de espécies pioneiras e das primárias suprimidas, resultando em um maior nível de diversidade em relação às florestas com dossel totalmente fechado. Porém, esse processo de sucessão secundária depende da habilidade competitiva das árvores das espécies e de sua tolerância às condições ambientais.

Portanto, pode-se observar que muitos bosques primários tropicais são produto de sucessões secundárias centenárias e, por isso, possuem um enorme potencial de manejo, ainda pouco explorado, face à pressão sobre as florestas primárias. Além disso, a vegetação secundária é parte integral da vida das comunidades da Amazônia (índios, ribeirinhos, seringueiros) desde milhares de anos, como fonte de alimento, medicina e matéria prima de vários produtos (NEBEL; MELBY, 2005).

Nas últimas décadas a Amazônia sofreu um desflorestamento predatório, pela crescente demanda de madeiras tropicais e, para evitar a extinção das espécies arbóreas, preconiza-se a aplicação do manejo florestal como tecnologia fundamental para o desenvolvimento sustentável da região. Porém, verifica-se a necessidade de suporte científico para o uso do manejo florestal de forma sustentável, com a necessidade de metodologia que estabeleça o diâmetro mínimo de corte (DMC) e de outros parâmetros relacionados com a dinâmica da floresta, como o crescimento das árvores (produção volumétrica), a mortalidade e o ingresso de novas plantas pela regeneração natural (WALKER; CHAPIN, 1987; WELDEN et al., 1991).

Portanto, a dendrocronologia, aliada a outros métodos (ex.: monitoramento em parcelas permanentes; técnica de modelagem de simulação da sucessão florestal e inferência dos padrões de desenvolvimento de distintos sítios em diferentes estágios de desenvolvimento), pode ser aplicada no estudo da dinâmica das florestas exploradas pelo manejo florestal associando-se às técnicas apropriadas de extração dos produtos madeireiros e não-madeireiros, com benefício econômico-ambiental para as comunidades locais (SCHÖNGART, 2008; BRIENEN, 2005).

As árvores de cedro (Cedrela sp.) apresentam troncos altos, madeira de fácil trabalhabilidade e acabamento, incluindo o seu polimento, ressaltando sua beleza, com possibilidade de substituir a madeira de mogno (Swietenia macrophylla King), com valor comercial mais elevado. Ainda, pela excelente qualidade da sua madeira, as árvores de cedro estão ameaçadas de extinção pela sua exploração seletiva (ALMEIDA et al., 2010).

O lenho das árvores de Cedrela sp. caracteriza-se pela formação de anéis de crescimento distintos na sua seção transversal, visíveis a olho nu e demarcados pelo parênquima marginal e pela disposição dos vasos em anéis semi-porosos, com diferença marcante no diâmetro dos vasos do lenho inicial e tardio tendo, portanto, grande potencial para aplicação em dendrocronologia (TOMAZELLO FILHO et al., 2000; ROIG, 2000).

Pelo exposto, o presente trabalho tem como objetivo determinar a idade e o incremento periódico do lenho no tronco das árvores nativas de Cedrela sp., em diferentes condições de crescimento (sítios e microsítios) para propiciar subsídios técnicos ao manejo florestal sustentável no estado do Acre. 


\section{MATERIAL E MÉTODOS}

\section{Descrição dos sítios e caracterização das árvores selecionadas}

As árvores de Cedrela sp. foram selecionadas em três sítios: Reserva Experimental Catuaba (REC), Fazenda São Jorge (F. S. Jorge) e Parque Zoobotânico (PZ), sendo que a REC foi subdivida em 3 microssítios (i) floresta primária (indicativa do clímax regional e sem registro histórico ou evidência de corte de árvores e de queimada), (ii) borda da mata (localizada até $10 \mathrm{~m}$ do entorno da estrada principal da REC) e (iii) pastagem (área particular no entorno da REC, desmatada no início da década de 70). Na Fazenda São Jorge foram selecionadas 23 árvores de Cedrela sp., sendo 14 árvores exploradas (árvores com DAP acima do diâmetro mínimo de corte, DAP> $50 \mathrm{~cm}$ ) e 9 árvores remanescentes (árvores com o diâmetro abaixo do nível de corte, DAP $<50 \mathrm{~cm}$ ); no Parque Zoobotânico foram selecionadas aleatoriamente 9 árvores de Cedrela sp. (Figuras 1 e 2, Tabela 1).

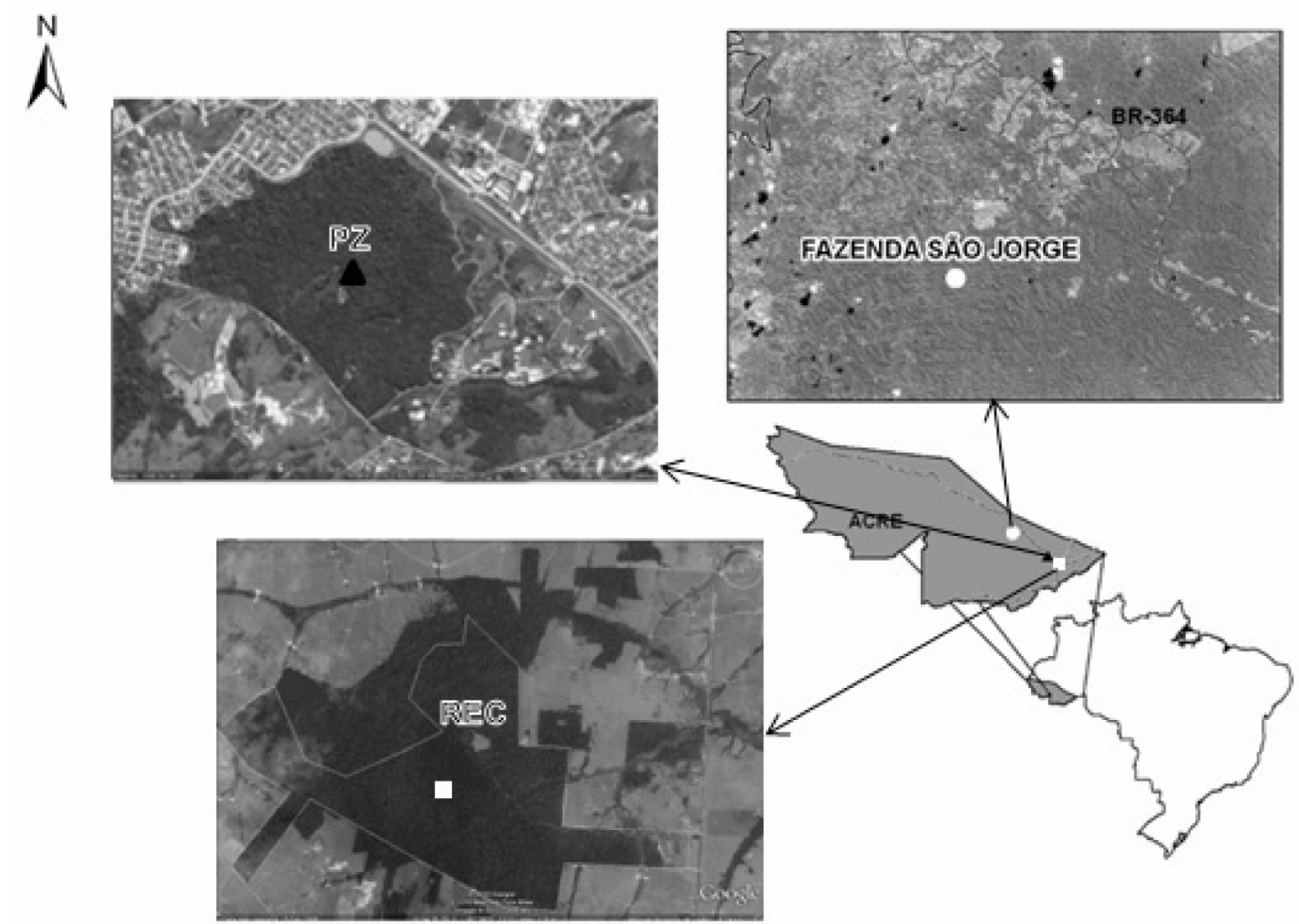

Figura 1. Mapa das áreas estudadas: Reserva Experimental Catuaba (REC), Parque Zoobotânico (PZ) e Fazenda São Jorge (FSJ).

Figure 1. Map of the study areas: Experimental Reserve Catuaba (REC), Zoobotanic Park (PZ) and Farm São Jorge (FSJ).

\section{Contagem e mensuração dos anéis de crescimento}

Aplicou-se metodologia específica (COOK; KAIRIUKSTIS, 1989) para determinação das séries de anéis de crescimento das árvores de Cedrela sp., após a realização do controle de qualidade para a detecção de anéis de crescimento falsos e faltantes utilizando-se o programa COFECHA. Na sequência foi aferido o número de anéis de crescimento formado no lenho do tronco das árvores de Cedrela sp. nas áreas de estudo, considerando a data da extração das amostras do lenho. Para a determinação do crescimento radial anual do tronco das árvores foram utilizados os valores médios da largura dos anéis de crescimento.

\section{Similaridade dendrocronológica entre os ambientes}

O agrupamento das árvores de Cedrela sp. foi feito pela determinação das diferentes condições de crescimento (sítios e microssítios), aplicando as técnicas de análise multivariada, com o software Statistica 7.0. Os resultados dos parâmetros dendrocronológicos (número e espessura dos anéis de crescimento e respectivo desvio padrão, além do DAP do tronco das árvores) foram submetidos a uma análise de dissimilaridade (distância euclidiana), obtendo-se os diferentes agrupamentos. Em seguida, os dados foram submetidos ao processo de estandarização, pela divisão dos valores de cada 
variável pelo valor do desvio padrão no conjunto de todas as amostras do lenho analisadas, retirando-se o efeito das diferenças de unidades das variáveis (REIS; RIBEIRO JR., 2010). Posteriormente, os dados foram submetidos à análise de componentes principais (ACP), analisando-se as covariâncias e as correlações baseadas nas raízes (ou valores) das variáveis e em seus vetores, considerando as duas primeiras raízes extraídas, por apresentarem os maiores autovalores (BORCARD et al., 2011).
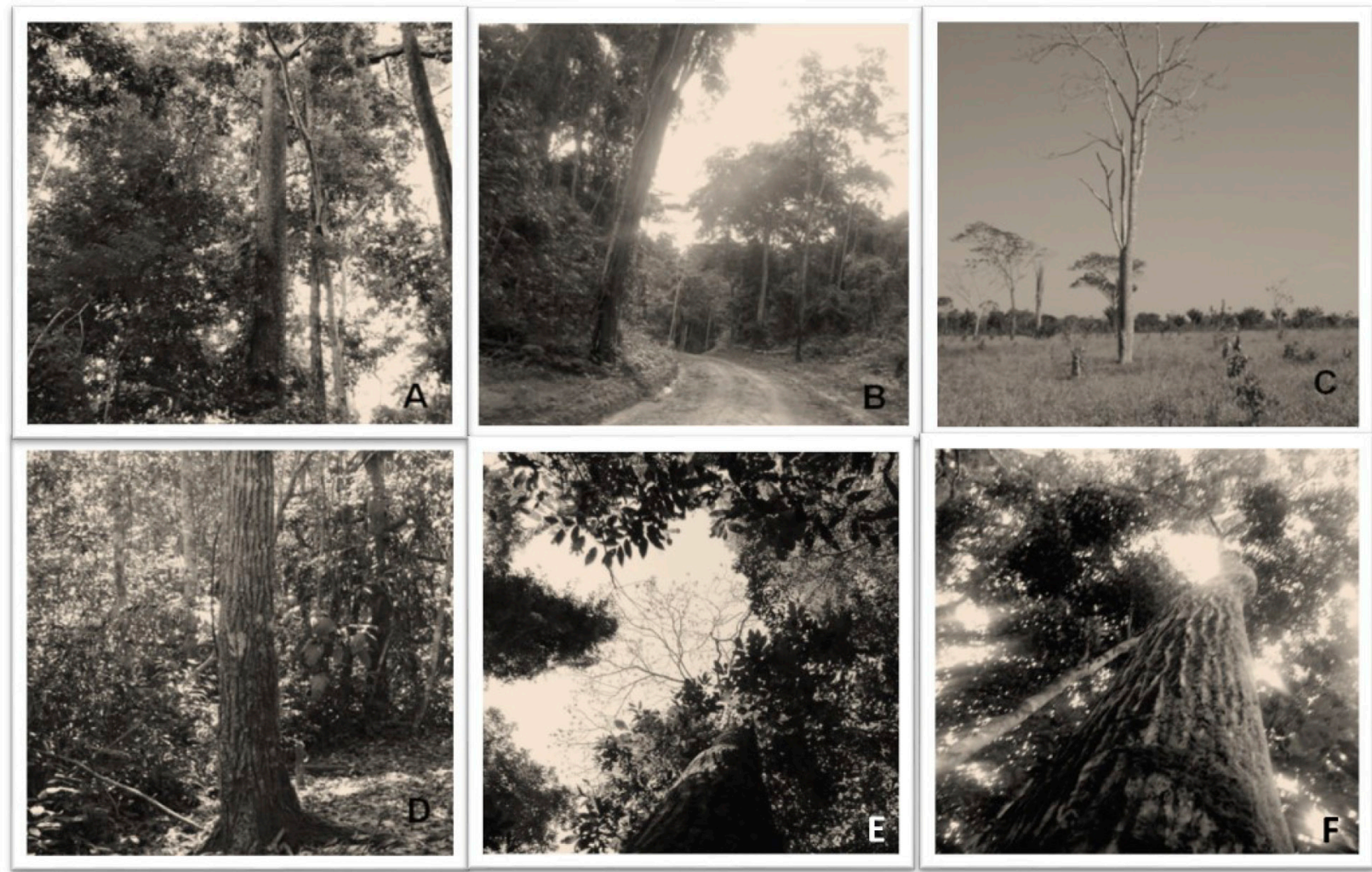

Figura 2. Microsítios da floresta primária (A), borda de floresta (B), pastagem (C) no sítio da Reserva Experimental Catuaba), floresta secundária (D) no Sítio do Parque Zoobotânico e floresta primária com árvore remanescente, (E) árvore de Cedrela sp. a ser explorada (F) na Fazenda São Jorge.

Figure 2. Microsites of primary forest (A), forest edge (B), pasture (C) at the site of Experimental Reserve Catuaba; secondary forest (D) in the Zoobotanic Park site and primary forest with remaining tree (E) and Cedrela sp. tree to harvested $(F)$ in Farm São Jorge.

\section{RESULTADOS E DISCUSSÃO}

\section{Distribuição do diâmetro do tronco das árvores}

O DAP das árvores de Cedrela sp. da REC em áreas de borda de mata e na floresta primária foi de 20,1 a 49,7 cm (DAP médio de 29,6 cm) e de 34,8 a 57,1 cm (DAP médio de 47,9 cm), respectivamente. O DAP do tronco das árvores de Cedrela sp. localizadas na área de pastagem da REC variou de 43,9 a 74,2 cm (DAP médio de 52,4 cm), valores bastante superiores aos DAPs encontrados nos ambientes de borda de mata e de floresta primária da REC (Tabela 1). No PZ, o DAP do tronco das árvores de Cedrela sp. ficou abaixo de $50 \mathrm{~cm}$, variando de 26,1 a 50,8 cm (DAP médio de $38,3 \mathrm{~cm}$ ) (Tabela 1).

Na FSJ, o DAP do tronco das árvores de Cedrela sp. exploradas foi superior a $50 \mathrm{~cm}$ (variação de 53,0 a $134,8 \mathrm{~cm}$; média de $71,6 \mathrm{~cm}$ ), a exceção da árvore $\mathrm{n}^{\circ} 14$, com $38,5 \mathrm{~cm}$, incluída no estudo, após a sua queda natural. As árvores de Cedrela sp. remanescentes tinham DAP do tronco de 28,3 a $55,3 \mathrm{~cm}$ (média de 35,6 cm), com a sua maioria não atingindo o diâmetro mínimo do tronco para o corte de $50 \mathrm{~cm}$ (Tabela 1).

$\mathrm{Na}$ análise da distribuição do diâmetro do tronco das árvores de Cedrela sp., nos diferentes ambientes, verifica-se nas áreas de pastagem a maior parte das árvores com diâmetro do tronco maior do que $50 \mathrm{~cm}$, indicando a ocorrência de condições favoráveis para o seu crescimento, com competição reduzida por água, nutrientes e luminosidade. Já nas áreas de borda a classe mais frequente de DAP do tronco das árvores de Cedrela sp. foi de $20-30 \mathrm{~cm}$ devido, provavelmente, a menor idade das árvores (22 anos em média) em relação às árvores da floresta primária (56 anos em média) (Tabela 1). 
Tabela 1. Valor médio do número e da largura dos anéis de crescimento e desvio padrão e o diâmetro a altura do peito (DAP) das árvores de Cedrela sp nos diferentes sítios e ambientes.

Table 1. Average number and width of growth rings and standard deviation and diameter at breast height (DBH) of Cedrela sp trees in different sites and environments.

\begin{tabular}{lccccccc}
\hline Sítio /ambiente & $\begin{array}{c}\mathbf{N}^{\circ} \text { de } \\
\text { árvores }\end{array}$ & $\begin{array}{c}\text { Larg. } \\
\text { anéis } \\
(\mathbf{m m})\end{array}$ & $\begin{array}{c}\mathbf{N}^{\circ} \text { de } \\
\text { anéis }\end{array}$ & $\begin{array}{c}\text { Desv } \\
\text { pad larg. } \\
\text { anéis }\end{array}$ & $\begin{array}{c}\text { DAP } \\
\text { médio } \\
(\mathbf{c m})\end{array}$ & $\begin{array}{c}\text { DAP } \\
\text { mínimo } \\
(\mathbf{c m})\end{array}$ & $\begin{array}{c}\text { DAP } \\
\text { máximo } \\
(\mathbf{c m})\end{array}$ \\
\hline REC /Borda de mata & 10 & 8,2 & 22 & 4,0 & 29,6 & 20,1 & 49,7 \\
REC/ Floresta 1a & 10 & 3,0 & 56 & 2,0 & 47,9 & 34,8 & 57,1 \\
REC/Pastagem & 10 & 4,9 & 44 & 3,0 & 52,4 & 43,9 & 74,2 \\
PZ /Floresta 2 $^{\text {a }}$ & 9 & 5,5 & 32 & 2,7 & 38,3 & 26,1 & 50,8 \\
FSJ/Floresta 1 $^{\text {a }}$ (exploradas) & 14 & 3,5 & 98 & 2,7 & 71,6 & $38,5(53,0)^{*}$ & 134,8 \\
FSJ/Floresta 1 $^{\text {a }}$ (remanescentes) & 9 & 2,2 & 68 & 1,5 & 35,6 & 28,3 & 55,3 \\
\hline
\end{tabular}

*Excluindo-se a árvore 14 que sofreu queda natural.

No PZ, o DAP do tronco das árvores de Cedrela sp. ficou abaixo de $50 \mathrm{~cm}$ próximo ao obtido para as árvores de Cedrela sp da borda de mata da REC devido ao fato de que essas áreas, em período recente, foram afetadas por perturbações antrópicas de extração vegetal, agricultura e pecuária (MORATO, 2004; MENESES-FILHO et al., 1995) (Tabela 1).

Na FSJ, o DAP médio do tronco das árvores de Cedrela sp. exploradas foi de 71,6 cm, e, nas árvores remanescentes de Cedrela sp. foi de $35,6 \mathrm{~cm}$. A grande heterogeneidade observada no DAP do tronco das árvores de Cedrela sp. indica a existência de significativa variação de sua idade e das condições de crescimento de cada sítio e microsítio (NUTTO, 2001) (Tabela 1).

\section{Número e largura dos anéis de crescimento}

As árvores de Cedrela sp. ocorrentes na área de borda de floresta apresentaram, em média, 22 anéis de crescimento com 8,2 mm de largura, as da floresta primária da REC apresentaram 56 anéis de crescimento, com $3 \mathrm{~mm}$ de largura, as da área de pastagem, com 44 anéis de crescimento com 4,9 $\mathrm{mm}$ de largura e as árvores da floresta secundária do PZ apresentaram, em média, 32 anéis de crescimento, com $2,7 \mathrm{~mm}$ de largura.

Em relação ao número e largura dos anéis de crescimento das árvores de Cedrela sp. da FSJ observa-se na Tabela 1, uma diferença marcante da largura média dos anéis de crescimento entre as árvores exploradas $(3,5 \mathrm{~mm})$ e as remanescentes $(2,2 \mathrm{~mm})$, mostrando que, provavelmente, as condições desiguais de competição, principalmente por luminosidade, foi determinante para que as árvores exploradas atingissem o diâmetro mínimo de corte determinado pela legislação, em um tempo menor do que as árvores remanescentes (CUNHA; FINGER, 2013).

Segundo Kartawinata et al. (1989), as árvores que permanecem nas populações florestais manejadas em exploração seletiva em ciclos de corte são, geralmente, de menor porte e geneticamente inferiores; sendo assim, este tipo de exploração de floresta nativa resulta predominantemente em árvores de qualidade inferior, com risco de perda da variabilidade genética e com o comprometimento das gerações futuras e, mesmo de extinção de espécies. Por outro lado, se a redução do crescimento do tronco das árvores remanescentes de Cedrela sp. apresentar relação com os fatores do ambiente pode-se recomendar a aplicação de práticas silviculturais (corte de lianas, raleamento, etc) liberando o seu crescimento (SOUZA; JARDIM, 1993). Desta forma, dever ser realizados estudos direcionados para a avaliação da variabilidade genética, características reprodutivas e demográficas das árvores das espécies mais exploradas em regime de manejo nas florestas naturais, incluindo aspectos do processo de sucessão e da dinâmica do crescimento das árvores.

\section{Similaridade dendrocronológica entre os ambientes}

A análise dos parâmetros dos anéis de crescimento - número, largura e desvio padrão e o DAP das árvores de Cedrela sp. ocorrentes nos sítio e ambientes - possibilitou agrupar as cronologias pela sua similaridade (Figuras 3A, 3B). Considerando os parâmetros dendrocronológicos e pela estandarização dos dados - retirando o efeito das diferenças de unidades existentes entre as variáveis verifica-se que as árvores de Cedrela sp. nos diferentes ambientes e sítios constituem grupos distintos (Tabela 2 e Figuras 3A e 3B). 
Lobão et al. - Análise de similaridade de árvores Cedrela sp. sob

diferentes condições de crescimento no leste do estado do Acre, Brasil

Tabela 2. Valores médios e desvio padrão do conjunto de variáveis dendrocronológicas utilizadas nas análises de Cluster.

Table 2. Average values and standard deviation of the dendrochronological variable group used in the Cluster analyses.

\begin{tabular}{lccc}
\hline Sitio/Ambiente & Média & Desvio padrão & Grupo \\
\hline FSJ /Floresta 1 & (exploradas) & 49,16 & Grupo 1 \\
REC /Floresta 1a & 44,69 & 29,09 & Grupo 2 \\
FSJ /Floresta 1 $^{\text {a }}$ (remanescentes) & 27,35 & 33,17 & Grupo 2 \\
REC./Pastagem & 27,86 & 24,74 & Grupo 3 \\
PZ /Floresta 2 & 25,15 & 17,81 & Grupo 4 \\
REC./Borda & 19,27 & 11,95 & Grupo 4 \\
\hline
\end{tabular}
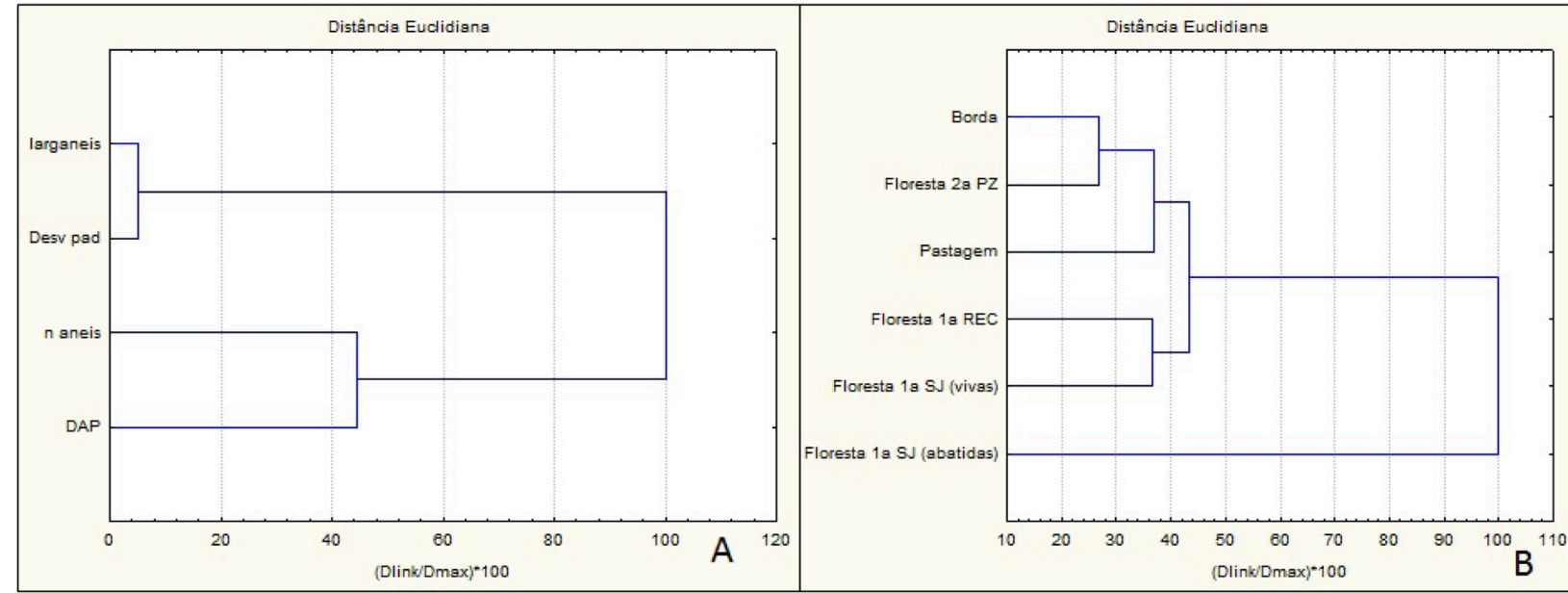

Figura 3. Dendrogramas de dissimilaridade (distância Euclidiana, em \%) das árvores de Cedrela sp.: variáveis dendrocronológicas (A) e espécies/sítios/ambientes (B).

Figure 3. Dendrograms of dissimilarity (Euclidean distance, in \%) of Cedrela sp. trees: dendrochronological variables (A) and species/sites/environments (B).

As árvores exploradas de Cedrela sp. da FSJ constituem um grupo diferenciado dos demais pela Distância Euclidiana igual a 100\%; verifica-se, posteriormente, a formação de 2 grupos separados pela Distância Euclidiana de 45\%, representativos das árvores de Cedrela sp. em ambiente de floresta primária (sítios de FSJ e REC) e as árvores dos demais ambientes que sofreram ação antrópica. Este último apresentou mais uma divisão por uma Distância Euclidiana de 37\%, separando-se o microssítio de pastagem dos demais ambientes antropizados. Por fim, houve a separação entre os conjuntos de árvores de Cedrela sp. (Borda da REC e Floresta $2^{\text {a }}$ do PZ) separados por uma Distância Euclidiana de 27\% (Figura 3B).

O conjunto de árvores de Cedrela sp. das florestas primárias se separou das árvores dos ambientes antropizados pelo maior DAP do tronco e pelo número de anéis de crescimento (idade); a separação das árvores de Cedrela sp. das florestas primárias da REC e de FSJ (árvores remanescentes) em relação às árvores exploradas da floresta primária de FSJ deve-se, principalmente, ao fato destas árvores (floresta 1a , FSJ) apresentarem maior DAP do tronco e maior largura dos anéis de crescimento (Figura 3A ).

Por outro lado, a separação do grupo de árvores de Cedrela sp. nos ambientes de borda e floresta $2^{\text {a }}$ do $\mathrm{PZ}$, do conjunto de árvores de Cedrela sp. da pastagem foi resultado, principalmente, da maior largura e menor número de anéis de crescimento (idade) (Figura 3A).

A análise de componentes principais aplicada nas árvores de Cedrela sp nos diferentes sítios e microssítios mostrou que as características dendrométricas resumem-se em 2 componentes, os quais, explicam 96,65\% (Tabela 3).

Foi determinada a importância das características dendrométricas das árvores nos dois componentes principais, através dos seus autovetores, permitindo-se estabelecer que: i) no componente principal 1 as características dendrométricas referentes à largura e ao número de anéis de crescimento, foram estatisticamente significativas, com este componente explicando $68,47 \%$ da variância total; ii) no componente principal 2 o DAP do tronco das árvores foi altamente significativo com o desvio padrão da largura dos anéis de crescimento, explicando $28,18 \%$ da variação dos dados (Tabelas 3 e 4 ). 

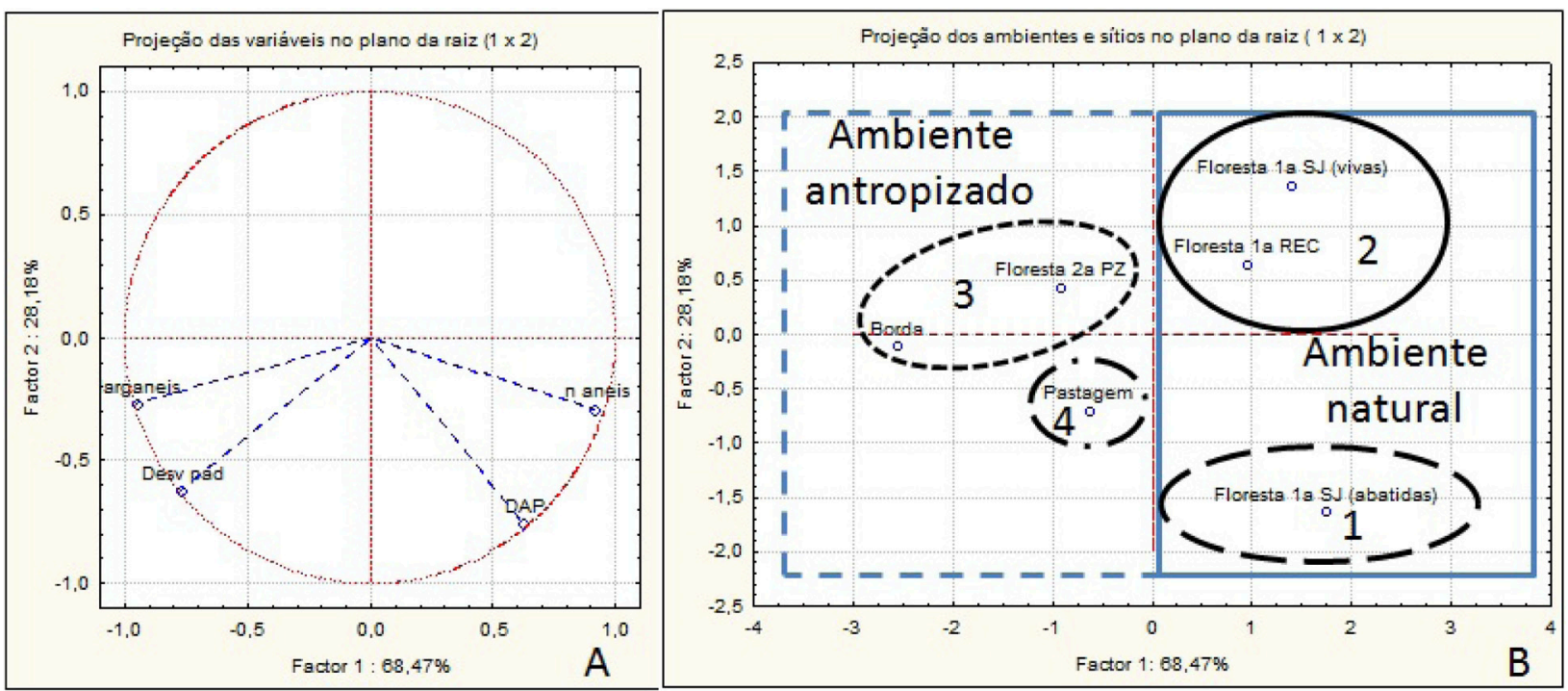

Figura 4. Análise de componentes principais das árvores de Cedrela sp.: variáveis dendrocronológicas (A) e espécies/sítios/ambientes (B).

Figure 4. Principal component analysis of Cedrela sp. trees: dendrochronological variables (A) and species/sites/ environments (B).

Tabela 3. Auto-valores, variância e valores acumulados da análise de componentes principais do conjunto de variáveis dendrocronológicas.

Table 3. Eigenvalues, variance and accumulated values of the principal component analysis of the dendrochronological variables.

\begin{tabular}{lcccc}
\hline Fator & Auto-valores (Eigenvalue) & Total da variância (\%) & Auto-valores acumulados & Variância acumulada (\%) \\
\hline 1 & 2,73 & 68,47 & 2,73 & 68,47 \\
2 & 1,13 & 28,18 & 3,86 & 96,65 \\
3 & 0,10 & 2,97 & 3,96 & 99,62 \\
4 & 0,04 & 0,38 & 4,00 & 100,00 \\
\hline
\end{tabular}

Tabela 4. Contribuição das variáveis dendrocronológicas baseadas nas correlações geradas pela Análise de Componentes Principais.

Table 4. Dendrochronological variables contribution based on correlations generated by Principal Component Analysis.

\begin{tabular}{lcc}
\hline Variáveis & Fator 1 & Fator 2 \\
\hline Largura dos anéis de crescimento & 0,33 & 0,07 \\
Número de anéis de crescimento (idade) & 0,30 & 0,08 \\
Desvio padrão(largura dos anéis de crescimento) & 0,22 & 0,35 \\
DAP (Diâmetro à Altura do Peito) & 0,14 & 0,51 \\
\hline
\end{tabular}

Os resultados da análise do fator 1 indicam que o conjunto das árvores dos ambientes antropizados em relação às florestas $1^{\text {as }}$ (naturais), possui uma maior largura de anéis de crescimento devido à menor competição por água, nutrientes e principalmente por luz com outras espécies arbóreas, e menor idade pela supressão de sua vegetação (Figura 4B).

Os resultados da análise do fator 2 indicam que o grupo das árvores de Cedrela sp. (exploradas) na floresta $1^{\mathrm{a}}$ da Fazenda São Jorge diferencia-se das árvores dos demais conjuntos, pelos maiores DAP's e maior número de anéis de crescimento (idade) (Tabela 4 e Figuras 4A e 4B). Foi, ainda, possível separar um segundo grupo de árvores de Cedrela sp. (remanescentes) da floresta $1^{\mathrm{a}}$ da Fazenda São Jorge e da floresta $1^{a}$ da REC por estes agrupamentos possuírem árvores com maior número de anéis de crescimento (idade) que as árvores dos ambientes antropizados (Figuras 4A e 4B).

Um terceiro grupo é formado pelo conjunto de árvores de Cedrela sp. de borda de floresta da REC e da floresta $2^{a}$ do PZ por estas possuírem maior largura dos anéis de crescimento, pouca idade e menores DAP's (Figura 4B).

Por fim, verifica-se que as árvores de Cedrela sp. de pastagem formaram um grupo isolado por apresentarem valores médios de largura, número de anéis de crescimento (idade) e DAP's relativamente altos (Figura 4B). 
Verifica-se que os métodos dendrocronológicos possuem grande potencial no manejo florestal sustentável para a definição dos ciclos de corte recomendando-se, porém, utilizar outros métodos diretos, como a determinação da periodicidade e da taxa de crescimento do tronco das árvores, em parcelas de inventário permanente, além de incorporar outros parâmetros. Dentre estes parâmetros, destacam-se a produtividade em biomassa $\left(\mathrm{m}^{3} / \mathrm{ha} / \mathrm{ano}\right)$, restrição do corte de árvores de determinadas espécies, definição do Diâmetro Mínimo de Corte (DMC) de cada espécie, sendo necessários estudos de dendrocronologia, de determinação da distribuição diamétrica do tronco das árvores, suas características ecológicas, frutificação, DAP máximo, uso da madeira, etc. conforme indicado por Gauto (1996).

\section{CONCLUSÕES}

Os resultados da análise dendrocronológica das árvores de Cedrela sp. permitem concluir que (i) a metodologia de contagem e de mensuração dos anéis de crescimento no lenho das árvores de Cedrela sp. demonstrou ser uma importante ferramenta para estudar a dinâmica da floresta (ii) as diferentes condições de crescimento (sítios e ambientes) influenciaram significativamente no crescimento radial do tronco das árvores de Cedrela sp.; (iii) na área de exploração florestal da Fazenda S. Jorge, as árvores exploradas $(\mathrm{DMC}>50 \mathrm{~cm})$ e as remanescentes mostram diferenças significativas no incremento em diâmetro do tronco, (iv) estas informações são importantes e podem ser incorporadas nas metodologias tradicionais para a definição de ciclos de corte e de produtividade em biomassa $\left(\mathrm{m}^{3} / \mathrm{ha} / \mathrm{ano}\right)$ das espécies florestais comercialmente exploradas.

\section{REFERÊNCIAS BIBLIOGRÁFICAS}

ALMEIDA, A. N.; ANGELO, SILVA, J. G. L.; HOEFLICH, V. A. Mercado de madeiras tropicais: substituição na demanda de exportação. Acta Amazônica, Manaus, v. 40 n. 1 p. 119-126. 2010.

ASQUITH, N. M. La dinámica del bosque y la diversidad arbórea. In: GUARIGUATA, M. R.; KATTAN, G. (Ed.) Ecología y conservación de bosques neotropicales. Cartago: Libro Universitario Regional, 2002. p. 377-406.

BORCARD, D., GILLET, F. E LEGENDRE, P. Numerical ecology with R. Springer. New York: Springer-Verlag, 2011, $306 \mathrm{p}$

BRIENEN, R. J. W. Tree rings in the tropics: a study on growth and ages of Bolivian rain forest trees. Riberalta: PROMAB, 2005. 144 p. (Scientific Series, 10).

BUSH, M. B. Amazonian speciation: a necessarily complex model. Journal of Biogeography, Oxford, v. 21, n. 1, p. 5-17, 1994.

COOK, E. R.; KAIRIUKSTIS, L. Methods of dendrochronology: application on the environmental sciences. Dordrecht: Kluwer Academic, 1989. 394 p.

COLINVAUX, P. A.; OLIVEIRA, P. E. ; MORENO, J. E.; MILLER, M. C.; BUSH, M. B. A. Pollen record from lowland Amazonia: forest and cooling in glacial times. Science, Washington, v. 274, n. 5284, p. 85-88, 1996.

CUNHA, T. A. D.; FINGER, C. A. G. Asymmetric competition and the diameter increment of Cedrela odorata L. trees in western Amazonia. Acta Amazônica, Manaus, v. 43, n. 1, p. 9-18, 2013.

GAUTO, O. A.; GRANCE, L. A.; MAIOCCO, D. C.; FIGUEIREDO FILHO, A. Crecimiento de la masa del bosque nativo en la reserva forestal Guaraní (Misiones). Yvyraretá, Eldorado, v. 7, n. 7, p. 5-10, 1996.

KARTAWINATA, K.; JESSUP, T. C.; VAYDA, A. P. Exploitation in southeast Asia. In: LIETH, H.; WERGER, M. J. A. (Ed.). Ecosystems: biogeographical and ecological studies. Netherlands: Elsevier, 1989. v. 2, p. 591-610. 
KATTAN, G. H. Fragmentación: patrones y mecanismos de extinción de especies. In: GUARIGUATA, M. R.; KATTAN, G. (Ed.).Ecología y Conservación de Bosques Neotropicales. Cartago: Libro Universitario Regional, 2002. p. 561-590.

MENESES-FILHO, L. C. L; FERRAZ, P. A.; PINHA, J. F. M.; FERREIRA, L. A.; BRILHANTE, N. A. Comportamento de 24 espécies arbóreas tropicais madeireiras introduzidas no Parque Zoobotânico Rio Branco-Acre. Rio Branco: Universidade Federal do Acre, 1995, v.1. p. 77-81

MORATO, E. F. Efeito da sucessão florestal sobre a nidificação de vespas e abelhas solitárias. 2004. 128 p. Tese (Doutorado em Ecologia, Manejo e Conservação de Vida Silvestre) - Universidade Federal de Minas Gerais, 2004.

NEBEL G.; MEILBY, H. Growth and population structure of timber species in peruvian flood plains. Forest Ecology and Management, Amsterdan, v. 215, n. 1-3, p. 196-211, 2005.

NUTTO, L. Manejo do crescimento diamétrico de Araucaria angustifolia (Bert.) O. Ktze. baseado na árvore individual. Ciência Florestal, Santa Maria, v. 11, n. 2, p. 9-25, 2001.

REIS, G. M.; RIBEIRO JR., J. I. Ferramentas estatísticas básicas da qualidade - guia prático do R. Disponível em: $<$ http://www.estatisticanor.xpg.com.br $>$. Acesso em: 20 out. 2010.

ROIG, F. A. Dendrocronología en los bosques del Neotrópico: revisión y prospección futura. In: ROIG, F. A. (Ed.).Dendrocronología en América Latina. Mendoza: EDIUNC, 2000. p. 381-431.

SCHÖNGART, J. Growth-orientated logging (GOL): a new concept towards sustainable forest management in Central Amazonian várzea floodplains. Forest Ecology and Management, Amsterdan, v. 256, p.46-58, 2008.

SOUZA, A. L.; JARDIM, F. C. S. Sistemas silviculturais aplicados às florestas tropicais. Viçosa: SIF, 1993. 125p. (Documento SIF, 008).

TOMAZELLO FILHO, M.; BOTOSSO, P. C.; LISI, S. C. Potencialidade da família Meliaceae para dendrocronologia em regiões tropicais e subtropicais. In: ROIG, F.A. Dendrocronologia en América Latina. Mendoza: Universidad Nacional de Cuyo, 2000. p.381 - 431.

WALKER, L. R. E; CHAPIN, F. S. Interactions among process controlling successional change. Oikos, Copenhagen, v. 50, p.131-135, 1987.

WELDEN, C. W.; HEWETT, S. W.; HUBELL, S. P.; FOSTER, R. B. Sapling survival, growth and recruitment: relationship to canopy height in a neotropical forest. Ecology, Durham, v.71, p.35-50, 1991.

Recebido em 12/01/2015

Aceito para publicação em 07/07/2015

Sci. For., Piracicaba, v. 44, n. 109, p. 231-239, mar. 2016

DOI: dx.doi.org/10.18671/scifor.v44n109.22 